\title{
MODELING AND ANALYTICAL STUDY FOR FERROELECTRIC MATERIALS
}

\author{
Alexander Mielke* and Aida M. Timofte ${ }^{\dagger}$ \\ * Weierstraß-Institut für Angewandte Analysis und Stochastik \\ Mohrenstraße 39, 10117 Berlin \\ e-mail: mielke@wias-berlin.de,web page: http://www.wias-berlin.de/people/mielke/ \\ ${ }^{\dagger}$ Universität Stuttgart, Institut für Analysis, Dynamik und Modellierung \\ Pfaffenwaldring 57, 70569 Stuttgart \\ e-mail: timofte@wias-berlin.de
}

Keywords: Ferroelectric material model, existence of solutions, energetic formulation

\begin{abstract}
We discuss rate-independent engineering models for multi-dimensional behavior of ferroelectric materials. These models capture the non-linear and hysteretic behavior of such materials. We show that these models can be formulated in an energetic framework which is based on the elastic and the electric displacements as reversible variables and interior, irreversible variables like the remanent polarization. We provide quite general conditions on the constitutive laws which guarantee the existence of a solution. Under more restrictive assumptions we are also able to establish uniqueness results.
\end{abstract}

\section{INTRODUCTION}

Ceramic materials and single crystals showing ferroelectric behavior are being used in many applications in electronics and optics. A crystal is ferroelectric if it has a spontaneous polarization which can be reversed in sense or reoriented by the application of an electric field, larger than the coercive field. Reversal is also known as switching. A large number of applications of ferroelectric ceramics also exploit properties that are an indirect consequence of ferroelectricity, such as dielectric, piezoelectric, pyroelectric, and electro-optic properties. Piezoelectricity is the ability of certain crystalline materials to develop an electrical charge proportional to a mechanical stress. It was discovered by the Curie brothers in 1880 (see Wikipedia). Piezoelectric materials also show a converse effect, where a geometric strain (deformation) is produced on the application of a voltage. Converse piezoelectricity was mathematically deduced from fundamental thermodynamic principles by Lippmann in 1881 ( see Wikipedia). The permanent electric dipole moment possessed by all pyroelectric (polar) materials may, in certain cases, be reoriented by the application of an electric field. The above comments are meant to point out that ferroelectric crystals are necessarily both pyroelectric and piezoelectric. 
Ferroelectricity is a phenomenon which was discovered in 1921 (see [16]). The name refers to certain magnetic analogies, though it is somewhat misleading, as it has no connection with iron (ferrum) at all. Ferroelectricity has also been called Seignette electricity, as Seignette or Rochelle Salt (RS) was the first material found to show ferroelectric properties, such as a spontaneous polarization on cooling below the Curie point, ferroelectric domains, and a ferroelectric hysteresis loop. A huge leap in the research on ferroelectric materials came in the 1950's, leading to the widespread use of barium titanate $\left(\mathrm{BaTiO}_{3}\right)$ based ceramics in capacitor applications and piezoelectric transducer devices. Since then, many other ferroelectric ceramics including lead titanate $\left(\mathrm{PbTiO}_{3}\right)$, lead zirconate titanate (PZT), lead lanthanum zirconate titanate (PLZT), and relaxor ferroelectrics like lead magnesium niobate (PMN), have been developed and utilized for a variety of applications. With the development of ceramic processing and thin film technology, many new applications have emerged. The biggest uses of ferroelectric ceramics have been in areas such as dielectric ceramics for capacitor applications, ferroelectric thin films for non volatile memories, piezoelectric materials for medical ultrasound imaging and actuators, and electro-optic materials for data storage and displays.

The model proposed in Section 2 captures the hysteretic behavior of ferroelectrics, by keeping in the mean time the general perspective for treating multi-axial behavior and complex geometries. It is based on the rate-independent, three-dimensional models used in the engineering literature, see $[1,2,3,4,5,6,7,8,9]$. These models work in the framework of small deformations and the quasistatic approximation for the elastic and electrostatic equilibria. However, certain internal variables $Q$, like the remanent polarization, are history dependent by an activation threshold and, thus, lead to a rate-independent evolution process.

We show that, using as primary reversible variables the elastic displacement $u: \Omega \rightarrow \mathbb{R}^{d}$ and the electric displacement $D: \mathbb{R}^{d} \rightarrow \mathbb{R}^{d}$, the process can be written in an energetic formulation which is based on the stored-energy functional

$$
\mathcal{E}(t, u, D, Q)=\int_{\Omega} W(x, \boldsymbol{\varepsilon}(u), D, Q)+\alpha(x, \nabla Q) \mathrm{d} x+\int_{\mathbb{R}^{d} \backslash \Omega} \frac{1}{2 \epsilon_{0}}|D|^{2} \mathrm{~d} x-\langle\ell(t),(u, D)\rangle
$$

and a dissipation potential of the form

$$
\mathcal{R}(\dot{Q}(t))=\int_{\Omega} R(x, \dot{Q}(t, x)) \mathrm{d} x .
$$

Thus, we will be able to take advantage of the recently developed energetic approach to rateindependent models, see $[10,11,12]$ and the survey [13]. This energetic formulation was originally developed for shape-memory alloys in $[14,15]$, but is now shown to apply for many different rate-independent material models such as finite-strain elastoplasticity, damage, brittle fracture, delamination and vortex pinning in superconductors.

The theory is based on a purely static stability condition (S) and the energy balance (E) which 
have to hold for all $t \in[0, T]$ :

(S) $\mathcal{E}(t, u(t), D(t), Q(t)) \leq \mathcal{E}(t, \widehat{u}, \widehat{D}, \widehat{Q})+\mathcal{R}(\widehat{Q}-Q(t))$ for all $\widehat{u}, \widehat{D}, \widehat{Q}$

(E) $\left.\mathcal{E}(t, u(t), D(t), Q(t))+\int_{0}^{t} \mathcal{R}(\dot{Q}(s))\right) \mathrm{d} s$ $=\mathcal{E}(0, u(0), D(0), Q(0))-\int_{0}^{t}\langle\dot{\ell}(s),(u(s), D(s))\rangle \mathrm{d} s$.

The major advantage of the formulation is that it does involve neither derivatives of the constitutive functions $W, \alpha$ and $R$ nor derivatives of the solution $(u, D, Q)$, since the dissipation integral $\left.\int_{0}^{t} \mathcal{R}(\dot{Q}(s))\right) \mathrm{d} s$ can be reformulated as a total variation.

We employ the abstract existence result for $(\mathrm{S}) \&(\mathrm{E})$ from $[10,12,13]$, which is reported in Section 3, and apply it to our ferroelectric model at hand in Section 4. We provide conditions on the constitutive functions $W, \alpha$ and $R$ which allow us to prove existence of solutions for (S) \& (E) in suitable function spaces. In the last Section 5 we discuss the question of uniqueness, which leads to severe restrictions on the constitutive functions $W$ and $\alpha$.

\section{MODELING FOR FERROELECTRIC MATERIALS}

Here we give a general description of a class of time-dependent models for ferroelectric materials. Our class of models is stimulated by the engineering models from $[3,4,5,7,8]$. However, we will rephrase the theory there in such a way that it can be formulated in terms of two energetic functionals, namely the stored energy $\mathcal{E}$ and the pseudo-potential $\mathcal{R}$ for the dissipation. Thus, we will be able to take advantage of the recently developed energetic approach to rateindependent models, see [10,11, 12] and the survey [13].

The basic quantities in the theory are the elastic displacement field $u: \Omega \rightarrow \mathbb{R}^{d}$ and the electric displacement field $D: \mathbb{R}^{d} \rightarrow \mathbb{R}^{d}$. Here, the electric displacement is also defined outside the body, as interior polarization of a ferroelectric material generates an electric field $E$ and displacement $D$ in all of $\mathbb{R}^{d}$ via the static Maxwell equation in $\mathbb{R}^{d}$. Commonly, the polarization $P$ is used for modeling, it is defined via

$$
D=\epsilon_{0} E+P,
$$

where $\epsilon_{0}$ is the dielectric constant (or permetivity) in the medium surrounding the body $\Omega$. Our formulation stays with $D$, since it leads to a simple and consistent thermomechanical model.

In addition we use internal variables $Q: \Omega \rightarrow \mathbb{R}^{d_{Q}}$ which, for instance, may be taken as a remanent strain $\varepsilon_{\text {rem }}$ or a remanent polarization $P_{\text {rem }}$.

The stored-energy functional has the following form:

$$
\mathcal{E}(t, u, D, Q)=\int_{\Omega}(W(x, \varepsilon(u), D, Q)+\alpha(\nabla Q)) \mathrm{d} x+\int_{\mathbb{R}^{d} \backslash \Omega} \frac{1}{2 \epsilon_{0}}|D|^{2} \mathrm{~d} x-\langle\ell(t),(u, D)\rangle,
$$

where $W$ is the Helmholtz free energy and $\varepsilon(u)$ is the infinitesimal strain tensor given by

$$
\varepsilon(u)=\frac{1}{2}\left(\nabla u+\nabla u^{\top}\right) \in \mathbb{R}_{\mathrm{sym}}^{d \times d}:=\left\{\varepsilon \in \mathbb{R}^{d \times d}: \varepsilon=\varepsilon^{\top}\right\} .
$$

The nonlocal term $\alpha(x, \nabla Q)$ in $\mathcal{E}$ usually takes the form $\frac{k}{2}|\nabla Q|^{2}$ with $k>0$. This term 
penalizes rapid changes of the internal variable by introducing a length scale which determines the minimal width of the interfaces between domains of different polarization.

The external loading $\ell(t)$ depends on the process time $t$ and is usually given by

$$
\langle\ell(t),(u, D)\rangle=\int_{\mathbb{R}^{d}} E_{\text {ext }}(t, x) \cdot D(x) \mathrm{d} x+\int_{\Omega} f_{\text {vol }}(t, x) \cdot u(x) \mathrm{d} x+\int_{\Gamma_{\text {Neu }}} f_{\text {surf }}(t, x) \cdot u(x) \mathrm{d} a(x),
$$

where $E_{\text {ext }}, f_{\text {vol }}$ and $f_{\text {surf }}$ are applied, external fields.

For the dissipation potential $\mathcal{R}$ we take the very simple form

$$
\mathcal{R}(\dot{Q})=\int_{\Omega} R(x, \dot{Q}(x)) \mathrm{d} x
$$

where $R(x, \cdot): \mathbb{R}^{d_{Q}} \rightarrow[0, \infty)$ is a convex function which is positively homogeneous of degree 1. Note that the dissipation potential acts on the rate $\dot{Q}=\frac{\partial}{\partial t} Q$ of the internal variable only. The classical way to describe dissipation in ferroelectrics is a switching function in the from

$$
\Phi\left(x, X_{Q}\right) \leq 0 \quad \text { with } X_{Q}=\frac{\partial}{\partial Q} W-\operatorname{div}(\operatorname{D} \alpha(\nabla Q)) .
$$

This is equivalent to our dissipation potential $R$ by the relation

$$
R(x, \dot{Q})=\max \left\{\dot{Q} \cdot X_{Q}: \Phi\left(x, X_{Q}\right) \leq 0\right\} .
$$

To formulate the rate-independent evolution law we use the thermomechanically conjugated forces

$$
\sigma=\frac{\partial}{\partial \varepsilon} W \in \mathbb{R}^{d \times d}, \quad E=\left\{\begin{array}{ll}
\frac{\partial}{\partial D} W & \text { on } \Omega, \\
\frac{1}{\epsilon_{0}} D & \text { on } \mathbb{R}^{d} \backslash \Omega
\end{array}, \quad X_{Q} \in \mathbb{R}^{d_{Q}},\right.
$$

where $\sigma$ is the stress tensor and $E$ the electric field. The elastic equilibrium equation and the Maxwell equations read

$$
\begin{array}{ll}
-\operatorname{div} \sigma+f_{\mathrm{vol}}(t, \cdot)=0 & \text { in } \Omega, \\
\operatorname{div} D=0 \text { and } \operatorname{curl}\left(E-E_{\text {ext }}(t, \cdot)\right)=0 & \text { in } \mathbb{R}^{d},
\end{array}
$$

where curl $E$ is defined as $\nabla E-(\nabla E)^{\top}$ for general dimensions.

The evolution of $Q$ follows the following force balance law:

$$
0 \in \partial R(x, \dot{Q})+X_{Q}
$$

where $\partial R(x, \cdot)$ is the subdifferential of the convex function $R(x, \cdot)$.

We now want to rewrite these equations, at least formally, as equations in function spaces. For this purpose we introduce a suitable state space $\mathcal{Y}=\mathcal{F} \times \mathcal{Q}$ as follows. The space $\mathcal{F}$ contains the functions $u$ and $D$ and takes the form

$$
\mathcal{F}=\mathcal{H} \times \mathrm{L}_{\text {div }}^{2}\left(\mathbb{R}^{d}\right), \text { where } \mathrm{L}_{\text {div }}^{2}\left(\mathbb{R}^{d}\right):=\left\{\psi \in \mathrm{L}^{2}\left(\mathbb{R}^{d} ; \mathbb{R}^{d}\right): \operatorname{div} \psi=0\right\}
$$

and $\mathcal{H}$ is a closed affine subspace of $\mathrm{H}^{1}\left(\Omega ; \mathbb{R}^{d}\right)$. The space $\mathcal{Q}$ contains the internal state functions $Q$ and is taken to be $\mathrm{W}^{1, q_{Q}}\left(\Omega ; \mathbb{R}^{d_{Q}}\right)$ for a suitable $q_{Q}>1$. 
Using the well-known fact (cf. [17], Thm.1.4) that the total space $L^{2}\left(\mathbb{R}^{d} ; \mathbb{R}^{d}\right)$ decomposes in an orthogonal way into the two closed subspaces $\mathrm{L}_{\text {div }}^{2}\left(\mathbb{R}^{d}\right)$ and

$$
\mathrm{L}_{\text {curl }}^{2}\left(\mathbb{R}^{d}\right)=\left\{\psi \in \mathrm{L}^{2}\left(\mathbb{R}^{d} ; \mathbb{R}^{d}\right): \operatorname{curl} \psi=0\right\}
$$

we obtain the following result.

Proposition 2.1 Denote by $\mathrm{D}_{D} \mathcal{E}(t, u, D, Q)[\widehat{D}]$ the Gâteaux derivative of $\mathcal{E}$ in the direction $\widehat{D}$. Then, we have

$$
\left(\forall \widehat{D} \in \mathrm{L}_{\operatorname{div}}^{2}\left(\mathbb{R}^{d}\right): \mathrm{D}_{D} \mathcal{E}(t, u, D, Q)[\widehat{D}]=0\right) \Longleftrightarrow \operatorname{curl}\left(E-E_{\text {ext }}(t, \cdot)\right)=0 \quad \text { in } \quad \mathbb{R}^{d}
$$

Thus, we implement the Maxwell equations simply by choosing a suitable function space and the condition $\mathrm{D}_{D} \mathcal{E}(t, u, D, Q)=0$.

Similarly, the elastic equilibrium is obtained by $\mathrm{D}_{u} \mathcal{E}(t, u, D, Q)=0$. The dissipative force balance can also be rewritten in functional and thus the full problem may be written as

$$
\begin{gathered}
\mathrm{D}_{u} \mathcal{E}(t, u(t), D(t), Q(t))=0, \quad \mathrm{D}_{D} \mathcal{E}(t, u(t), D(t), Q(t))=0, \\
0 \in \partial \mathcal{R}(\dot{Q}(t))+\mathrm{D}_{Q} \mathcal{E}(t, u(t), D(t), Q(t)) .
\end{gathered}
$$

In fact, our theory is not based on the force balance (11). Instead, following [13, 14, 15], we use a weaker formulation which is based on energies only. This energetic formulation avoids derivatives of $\mathcal{E}$ and of the solution $(u, D, Q)$. Under suitable smoothness and convexity assumptions (see Section 5) the energetic formulation is equivalent to (11). We call $(u, D, Q)$ an energetic solution of the problem associated with $\mathcal{E}$ and $\mathcal{R}$, if for all $t \in[0, T]$ the stability condition (S) and the energy balance (E) hold:

$$
\begin{aligned}
\text { (S) } & \mathcal{E}(t, u(t), D(t), Q(t)) \leq \mathcal{E}(t, \widehat{u}, \widehat{D}, \widehat{Q})+\mathcal{R}(\widehat{Q}-Q(t)) \text { for all } \widehat{u}, \widehat{D}, \widehat{Q} ; \\
(\mathrm{E}) & \left.\mathcal{E}(t, u(t), D(t), Q(t))+\int_{0}^{t} \mathcal{R}(\dot{Q}(s))\right) \mathrm{d} s \\
& =\mathcal{E}(0, u(0), D(0), Q(0))-\int_{0}^{t}\langle\dot{\ell}(s),(u(s), D(s))\rangle \mathrm{d} s .
\end{aligned}
$$

Using the following abstract result, we will show that the energetic formulation (S) \& (E) has solutions for suitable initial data, if the constitutive functions $W, \alpha$ and $R$ satisfy reasonable continuity and convexity assumptions. Then, we will discuss stronger conditions which imply uniqueness.

\section{EXISTENCE OF SOLUTIONS IN THE GENERAL CASE}

We next recall the concept of energetic solution. Consider the set $\mathcal{Y}=\mathcal{F} \times \mathcal{Z}$ as the basic state space. Whenever possible, we will write $y$ instead of $(\varphi, z)$ to shorten notation. Note that the splitting is done such that changes in $z$ involve dissipation, whereas those of $\varphi$ do not. In the section above $(u, D)$ takes the rôle of $\varphi$ and $Q$ is the internal variable $z$.

The state space $\mathcal{Y}$ is equipped with a Hausdorff topology $\mathcal{T}=\mathcal{T}_{\mathcal{F}} \times \mathcal{T}_{\mathcal{Z}}$ and we denote by $y_{k} \stackrel{\mathcal{Y}}{\rightarrow} y, \varphi_{k} \stackrel{\mathcal{F}}{\rightarrow} \varphi$ and $z_{k} \stackrel{\mathcal{Z}}{\rightarrow} z$ the corresponding convergence of sequences.

The first ingredient of the energetic formulation is the dissipation distance $\mathcal{D}: \mathcal{Z} \times \mathcal{Z} \rightarrow$ $[0, \infty]$, which is a semi-distance (see (A1) below). For a given curve $z:[0, T] \rightarrow \mathcal{Z}$ we define 
the total dissipation on $[s, t]$ via

$$
\operatorname{Diss}_{\mathcal{D}}(z ;[s, t])=\sup \left\{\sum_{1}^{N} \mathcal{D}\left(z\left(\tau_{j-1}\right), z\left(\tau_{j}\right)\right): N \in \mathbb{N}, s=\tau_{0}<\tau_{1}<\cdots<\tau_{N}=t\right\} .
$$

The second ingredient is the energy-storage functional $\mathcal{E}:[0, T] \times \mathcal{Y} \rightarrow \mathbb{R}_{\infty}:=\mathbb{R} \cup\{\infty\}$. Here, $t \in[0, T]$ plays the rôle of a (very slow) process time which changes the underlying system via changing loading conditions. We assume that for all $y$ with $\mathcal{E}\left(t, y_{*}\right)<\infty$, the function $\mathbb{R} \ni t \mapsto \mathcal{E}\left(t, y_{*}\right) \in \mathbb{R}$ is differentiable.

Definition 3.1 A curve $y=(\varphi, z):[0, T] \rightarrow \mathcal{Y}=\mathcal{F} \times \mathcal{Z}$ is called an energetic solution of the rate-independent system associated with $(\mathcal{D}, \mathcal{E})$, if $t \mapsto \partial_{t} \mathcal{E}(t, y(t))$ is integrable and if the global stability (S) and the energy balance (E) hold for all $t \in[0, T]$ :

(S) For all $\widehat{y}=(\widehat{\varphi}, \widehat{z}) \in \mathcal{Y}$ we have $\mathcal{E}(t, y(t)) \leq \mathcal{E}(t, \widehat{y})+\mathcal{D}(z(t), \widehat{z})$.

(E) $\mathcal{E}(t, y(t))+\operatorname{Diss}_{\mathcal{D}}(z ;[0, t])=\mathcal{E}(0, y(0))+\int_{0}^{t} \partial_{t} \mathcal{E}(\tau, y(\tau)) \mathrm{d} \tau$.

To prove our existence result we impose the conditions (A1), (A2) and (A3) on the dissipation distance $\mathcal{D}$ :

$$
\begin{aligned}
& \text { (i) } \forall z_{1}, z_{2} \in \mathcal{Z}: \mathcal{D}\left(z_{1}, z_{2}\right)=0 \Longleftrightarrow z_{1}=z_{2}, \\
& \text { (ii) } \forall z_{1}, z_{2}, z_{3} \in \mathcal{Z}: \mathcal{D}\left(z_{1}, z_{3}\right) \leq \mathcal{D}\left(z_{1}, z_{2}\right)+\mathcal{D}\left(z_{2}, z_{3}\right) .
\end{aligned}
$$

Here, (i) is the classical positivity of a distance and (ii) the triangle inequality. Note that we allow the value $\infty$, and that we do not enforce symmetry, i.e., $\mathcal{D}\left(z_{1}, z_{2}\right) \neq \mathcal{D}\left(z_{2}, z_{1}\right)$ is allowed, as this is needed in many applications.

$$
\begin{aligned}
& \text { For any sequence }\left(z_{k}\right)_{k} \text { and any } z \text { in } \mathcal{Z} \text { we have: } \\
& \min \left\{\mathcal{D}\left(z_{k}, z\right), \mathcal{D}\left(z, z_{k}\right)\right\} \rightarrow 0 \text { for } k \rightarrow \infty \Longrightarrow z_{k} \stackrel{\mathcal{Z}}{\rightarrow} z \text { for } k \rightarrow \infty . \\
& \qquad \mathcal{D}: \mathcal{Z} \times \mathcal{Z} \rightarrow[0, \infty] \quad \text { is continuous. }
\end{aligned}
$$

For the energy functional $\mathcal{E}$ we impose the conditions (A4), (A5) and (A6):

$$
\mathcal{E}(t, \cdot): \mathcal{Y} \rightarrow \mathbb{R}_{\infty} \text { has compact sublevels } \forall t \in[0, T] .
$$

Here the sublevels $L_{t, e}$ of $\mathcal{E}(t, \cdot)$ are defined as usual by $L_{t, e}:=\{y \in \mathcal{Y}: \mathcal{E}(t, y) \leq e\}$.

$$
\begin{aligned}
& \text { There exist } c_{E}^{(1)}, c_{E}^{(0)}>0 \text { such that for all } y_{*} \in \mathcal{Y}: \\
& \text { If } \mathcal{E}\left(t, y_{*}\right)<\infty, \text { then } \partial_{t} \mathcal{E}\left(\cdot, y_{*}\right):[0, T] \rightarrow \mathbb{R} \text { is measurable } \\
& \quad \text { and }\left|\partial_{t} \mathcal{E}\left(t, y_{*}\right)\right| \leq c_{E}^{(1)}\left(\mathcal{E}\left(t, y_{*}\right)+c_{E}^{(0)}\right) . \\
& \forall E^{*}>0 \forall \varepsilon>0 \exists \delta>0: \quad \mathcal{E}(t, y) \leq E^{*} \text { and }|t-s| \leq \delta \\
& \\
& \Longrightarrow\left|\partial_{t} \mathcal{E}(t, y)-\partial_{t} \mathcal{E}(s, y)\right|<\varepsilon .
\end{aligned}
$$

The following existence result is proved in $[10,13]$.

Theorem 3.2 Assume that $\mathcal{E}$ and $\mathcal{D}$ satisfy the hypotheses (A1)-(A6) and that the initial datum $y_{0} \in \mathcal{Y}$ is stable (i.e., $y_{0}$ satisfies $(\mathrm{S})$ at $\left.t=0\right)$, then there exists a solution $y=(\varphi, z):[0, T] \rightarrow$ $\mathcal{Y}$ of $(\mathrm{S}) \&(\mathrm{E})$ with $y(0)=y_{0}$. 
Moreover, any solution of $(\mathrm{S}) \&(\mathrm{E})$ with $y(0)=y_{0}$ satisfies the a priori estimates

$$
\left.\begin{array}{c}
\mathcal{E}(t, y(t)) \leq\left(c_{E}^{(0)}+\mathcal{E}\left(0, y_{0}\right)\right) \mathrm{e}^{c_{E}^{(1)} t}-c_{E}^{(0)} \\
\operatorname{Diss}_{\mathcal{D}}(y ;[0, t]) \leq\left(c_{E}^{(0)}+\mathcal{E}\left(0, y_{0}\right)\right) \mathrm{e}^{c_{E}^{(1)} t}
\end{array}\right\} \quad \text { for } t \in[0, T] .
$$

\section{EXISTENCE THEOREM FOR FERROELECTRIC MODELS}

To apply the above abstract theory, we need the following standard result in linearized elasticity. It allows us to obtain the desired coercivity of the energy functional which is used to establish condition (A4).

Proposition 4.1 (Korn's inequality) Let $\Omega \subset \mathbb{R}^{d}$ be a nonempty connected open bounded set, with Lipschitz boundary $\Gamma$, and let $\Gamma_{\mathrm{Dir}}$ be a measurable subset of $\Gamma$, such that $\int_{\Gamma_{\mathrm{Dir}}} 1 \mathrm{~d} a>0$. Given a function $u \in \mathrm{H}^{1}\left(\Omega, \mathbb{R}^{d}\right)$, the linearized strain tensor $\varepsilon$ is defined by (3). Then, there exists a constant $k>0$, such that

$$
\|u\|_{\mathrm{H}^{1}}^{2} \leq k \int_{\Omega}|\varepsilon(u)|^{2} \mathrm{~d} x \quad \text { for all } u \in \mathrm{H}_{\Gamma_{\text {Dir }}}^{1}\left(\Omega, \mathbb{R}^{d}\right):=\left\{v \in \mathrm{H}^{1}\left(\Omega, \mathbb{R}^{d}\right):\left.v\right|_{\Gamma_{\text {Dir }}} \equiv 0\right\} .
$$

We now provide conditions on the constitutive functions $W, \alpha$ and $R$, such that the above abstract theory can be applied for our ferroelectricity model defined via the energy functional $\mathcal{E}$ in (2) and the dissipation potential $\mathcal{R}$ in (5).

The first assumption concerns the domain and the Dirichlet boundary:

$$
\Omega \text { and } \Gamma_{\text {Dir }} \text { satisfy the assumptions of Proposition } 4.1 \text {. }
$$

The function $R: \Omega \times \mathbb{R}^{d_{Q}} \rightarrow[0, \infty)$ satisfies

$$
\begin{gathered}
R \in \mathrm{C}^{0}\left(\bar{\Omega} \times \mathbb{R}^{d_{Q}}\right) \quad \text { and } \exists c_{R}, C_{R}>0 \forall V \in \mathbb{R}^{d_{Q}}: c_{R}|V| \leq R(x, V) \leq C_{R}|V| . \\
\forall x \in \Omega: R(x, \cdot): \mathbb{R}^{d_{Q}} \rightarrow[0, \infty) \text { is 1-homogeneous and convex. }
\end{gathered}
$$

The functions $W$ and $\alpha$ have to fulfill the following three conditions:

$$
W: \Omega \times \mathbb{R}_{\text {sym }}^{d \times d} \times \mathbb{R}^{d} \times \mathbb{R}^{d_{Q}} \rightarrow[0, \infty) \text { is a Caratheodory function, }
$$

which means that for each $(\varepsilon, D, Q)$ the function $W(\cdot, \varepsilon, D, Q)$ is measurable on $\Omega$ and for a.e. $x \in \Omega$ the mapping $W(x, \cdot, \cdot, \cdot)$ is continuous on $\mathbb{R}_{\mathrm{sym}}^{d \times d} \times \mathbb{R}^{d} \times \mathbb{R}^{d_{Q}}$.

$$
\begin{aligned}
& \exists c_{\mathcal{E}}, C_{\mathcal{E}}>0, q>1 \forall(x, \varepsilon, D, Q, V) \in \mathbb{R}_{\mathrm{sym}}^{d \times d} \times \mathbb{R}^{d} \times \mathbb{R}^{d_{Q}} \times \mathbb{R}^{d_{Q}}: \\
& W(x, \varepsilon, D, Q)+\alpha(V) \geq c_{\mathcal{E}}\left(|\varepsilon|^{2}+|D|^{2}+|Q|^{q}+|V|^{q}\right)-C_{\mathcal{E}} . \\
& \alpha: \mathbb{R}^{d_{Q} \times d} \rightarrow \mathbb{R} \text { is convex and } \\
& \forall(x, Q) \in \Omega \times \mathbb{R}^{d_{Q}}: W(x, \cdot, \cdot, Q): \mathbb{R}_{\mathrm{sym}}^{d \times d} \times \mathbb{R}^{d} \rightarrow \mathbb{R} \text { is convex. }
\end{aligned}
$$

For the external loading $\ell(t)$ we assume

$$
\ell \in \mathrm{C}^{1}\left([0, T],\left(\mathrm{H}_{\Gamma_{\mathrm{Dir}}}^{1}\left(\Omega ; \mathbb{R}^{d}\right)\right)^{*} \times \mathrm{L}_{\mathrm{div}}^{2}\left(\mathbb{R}^{d}\right)^{*}\right) .
$$


We now relate the concrete ferroelectric model to the abstract one by choosing the function spaces first:

$$
\mathcal{F}=\mathrm{H}_{\Gamma_{\text {Dir }}}^{1}\left(\Omega, \mathbb{R}^{d}\right)_{\text {weak }} \times \mathrm{L}_{\text {div }}^{2}\left(\mathbb{R}^{d}\right)_{\text {weak }} \quad \text { and } \quad \mathcal{Z}=\mathrm{L}^{1}\left(\Omega, \mathbb{R}^{d_{Q}}\right)_{\text {strong }} .
$$

Here the subscripts "weak" and "strong" indicate whether we use the weak or the strong (norm) topology in the corresponding Banach spaces. The dissipation distance $\mathcal{D}$ is related to $\mathcal{R}$ by

$$
\mathcal{D}\left(Q_{0}, Q_{1}\right)=\mathcal{R}\left(Q_{1}-Q_{0}\right)=\int_{\Omega} R\left(x, Q_{1}(x)-Q_{0}(x)\right) \mathrm{d} x .
$$

The functional $\mathcal{E}$ is defined on $[0, T] \times \mathcal{F} \times \mathcal{Z}$ via (2), where $\mathcal{E}(t, u, D, Q)$ takes the value $+\infty$, if $Q \notin \mathrm{W}^{1, q}\left(\Omega ; \mathbb{R}^{d_{Q}}\right)$, or if the integrand is not in $\mathrm{L}^{1}(\Omega)$. With these choices, the abstract energetic problem of Definition 3.1 leads us exactly to the energetic problem (S) \& (E) for the ferroelectric model as defined in (12).

Thus, our first main result will be proved by checking the assumptions of the abstract existence theorem 3.2 from above.

\section{Theorem 4.2 (Existence theorem)}

If the assumptions (B0)-(B6) hold, then for each stable initial condition $\left(u_{0}, D_{0}, Q_{0}\right) \in \mathcal{F} \times \mathcal{Z}$ the energetic problem $(\mathrm{S}) \&(\mathrm{E})$ in $(12)$ has a solution $(u, D, Q):[0, T] \rightarrow \mathcal{F} \times \mathcal{Z}$, with $(u(0), D(0), Q(0))=\left(u_{0}, D_{0}, Q_{0}\right)$, which satisfies

$$
(u, D, Q) \in \mathrm{L}^{\infty}\left([0, T] ; \mathrm{H}_{\Gamma_{\mathrm{Dir}}}^{1}\left(\Omega ; \mathbb{R}^{d}\right) \times \mathrm{L}_{\mathrm{div}}^{2}\left(\mathbb{R}^{d}\right) \times \mathrm{W}^{1, q}\left(\Omega ; \mathbb{R}^{d_{Q}}\right)\right) .
$$

\section{UNIQUENESS OF SOLUTIONS}

Uniqueness results in rate-independent hysteresis models are rather exceptional, as they need strong assumptions on the nonlinearities. We now show that suitable restrictions on our ferroelectric model leads to uniqueness of solutions. However, it is unclear whether these restrictions are still compatible with models which are useful in practice.

First of all, the theory has to be restricted to a Hilbert space setting, and we let

$$
\mathcal{Y}_{2}=\mathrm{H}_{\Gamma_{\text {Dir }}}^{1}\left(\Omega ; \mathbb{R}^{d}\right) \times \mathrm{L}_{\text {div }}^{2}\left(\mathbb{R}^{d}\right) \times \mathrm{H}^{1}\left(\Omega ; \mathbb{R}^{d_{Q}}\right) .
$$

We still assume that the conditions (B0)-(B6) hold, but now with $q=2$. We will add further condition below, such that we are able to apply the following abstract result [11, Thm. 7.4].

It is formulated on a general Hilbert space $\mathcal{Y}_{H}$ with functionals $\mathcal{E}:[0, T] \times Y_{H} \rightarrow \mathbb{R}$ and $\mathcal{R}: \mathcal{Y}_{H} \rightarrow \mathbb{R}$. The following conditions are imposed:

$$
\mathcal{R}: \mathcal{Y}_{H} \rightarrow[0, \infty) \text { is continuous, convex and 1-homogeneous. }
$$

$$
\begin{aligned}
& \mathcal{E} \in \mathrm{C}_{\text {loc }}^{2, \mathrm{Lip}}\left([0, T] \times \mathcal{Y}_{H}, \mathbb{R}\right) \text { and } \\
& \forall e_{0} \exists C>0 \forall y_{j} \text { with } \mathcal{E}(0, y) \leq e_{0}:\left\|\mathrm{D} \mathcal{E}\left(t, y_{1}\right)\right\|,\left\|\mathrm{D}^{2} \mathcal{E}\left(t, y_{1}\right)\right\| \leq C, \\
&\left\|\mathrm{D}^{2} \mathcal{E}\left(t, y_{1}\right)-\mathrm{D}^{2} \mathcal{E}\left(t, y_{2}\right)\right\| \leq C\left\|y_{1}-y_{2}\right\| . \\
& \exists \alpha>0 \forall v, y \in \mathcal{Y}_{H}:\left\langle\mathrm{D}^{2} \mathcal{E}(t, y) v, v\right\rangle \geq \alpha\|v\|^{2} .
\end{aligned}
$$


Theorem 5.1 If the conditions $(\mathrm{C} 1)-(\mathrm{C} 3)$ and $(\mathrm{A} 5)$ hold, then the energetic problem $(\mathrm{S}) \&(\mathrm{E})$ has for each stable initial datum $y_{0} \in \mathcal{Y}_{H}$ a unique solution $y:[0, T] \rightarrow \mathcal{Y}_{H}$ with $y(0)=y_{0}$. This solution satisfies $y \in \mathrm{C}^{\mathrm{Lip}}\left([0, T] ; \mathcal{Y}_{H}\right)$ and depends Lipschitz continuously on the initial data. Moreover, these solutions satisfy the differential inclusion

$$
0 \in \partial \mathcal{R}(\dot{y}(t))+\mathrm{D} \mathcal{E}(t, y(t)) \quad \text { for a.e. } t \in[0, T],
$$

which is equivalent to the quasi-variational inequality

$$
\left(\forall v \in \mathcal{Y}_{H}:\langle\mathrm{D} \mathcal{E}(t, y(t)), v-\dot{y}(t)\rangle+\mathcal{R}(\dot{y}(t)) \geq 0\right) \quad \text { for a.e. } t \in[0, T] .
$$

In our ferroelectric model the new assumptions $(\mathrm{C} 1)-(\mathrm{C} 3)$ can be satisfied only in the presence of the following additional conditions:

$$
\alpha(x, \nabla Q)=\frac{1}{2}\left\langle A_{Q}(x) \nabla Q, \nabla Q\right\rangle \quad \text { with } a_{0} \mathbf{1} \leq A_{Q}(x) \leq a_{1} \mathbf{1} \text { a.e. on } \Omega,
$$

where $a_{1} \geq a_{0}>0$.

$$
W(x, \varepsilon, D, Q)=\frac{1}{2}\left\langle\mathbb{A}(x)\left(\begin{array}{c}
\varepsilon-\varepsilon_{\mathrm{rem}}(x, Q) \\
D-P_{\mathrm{rem}}(x, Q)
\end{array}\right),\left(\begin{array}{c}
\varepsilon-\varepsilon_{\mathrm{rem}}(x, Q) \\
D-P_{\mathrm{rem}}(x, Q)
\end{array}\right)\right\rangle+W_{\text {hard }}\left(x, P_{\mathrm{rem}}\right),
$$

where $\mathbb{A}(x)=\left(\begin{array}{cc}A(x) & -B(x) \\ -B^{\top}(x) & C(x)\end{array}\right)$ is assumed to be bounded and uniformly positive definite on $\mathbb{R}_{\mathrm{sym}}^{d \times d} \times \mathbb{R}^{d}$.

$$
\begin{gathered}
W_{\text {hard }} \in \mathrm{L}^{\infty}\left(\Omega ; \mathrm{C}^{3}\left(\mathbb{R}^{d_{Q}}\right) \text { and } \exists C>0 \forall x, Q:\left|\mathrm{D}_{Q}^{3} W(x, Q)\right| \leq C(1+|Q|)^{d_{p}-3} .\right. \\
\quad\left(\varepsilon_{\text {rem }}, P_{\text {rem }}\right) \in \mathrm{L}^{\infty}\left(\Omega ; \mathrm{C}^{3}\left(\mathbb{R}^{d_{Q}} ; \mathbb{R}_{\text {sym }}^{d \times d} \times \mathbb{R}^{d}\right)\right), \quad p_{d} \geq 6 \text { and } \\
\exists C>0 \forall x, Q:\left|\mathrm{D}_{Q}^{3} \varepsilon_{\text {rem }}(x, Q)\right|,\left|\mathrm{D}_{Q}^{3} P_{\text {rem }}(x, Q)\right| \leq C(1+|Q|)^{\left(p_{d}-6\right) / 2} .
\end{gathered}
$$

Note that the restriction $p_{d}=2 d /(d-2) \geq 6$ leads to the restriction $d \leq 3$.

The final condition to be added is a uniform convexity on the sum of $W$ and $\alpha$ :

$$
\exists c_{0}>0 \forall x, \boldsymbol{\varepsilon}, D, Q: \mathrm{D}_{\boldsymbol{\varepsilon}, D, Q}^{2} W(x, \boldsymbol{\varepsilon}, D, Q) \geq c_{0} \mathbf{1} .
$$

Note that this condition is stronger than (B5), where convexity in $Q$ was not needed.

Theorem 5.2 Assume $d \in\{2,3\}$ and $\ell \in \mathrm{C}^{2}\left([0, T] ; \mathcal{F}_{*}\right)$. Moreover, let the conditions (B0)-(B4) with $q=2$ and the conditions (16)-(20) be satisfied. Then, the energetic formulation (S) \& (E) has for each stable initial datum $y_{0}=\left(u_{0}, D_{0}, Q_{0}\right)$ a unique solution $y=(u, D, Q)$ : $[0, T] \rightarrow \mathcal{Y}_{2}=\mathrm{H}_{\Gamma_{\text {Dir }}}^{1}\left(\Omega ; \mathbb{R}^{d}\right) \times \mathrm{L}_{\text {div }}^{2}\left(\mathbb{R}^{d}\right) \times \mathrm{H}^{2}\left(\Omega ; \mathbb{R}^{d_{Q}}\right)$

Moreover, other conclusions of Theorem 5.1 hold as well.

As a conclusion, we may say that it is possible to prove existence results for slight modifications of the engineering models. However, for the presently developed uniqueness theory the conditions are very restrictive and seem to contradict most useful models. 


\section{REFERENCES}

[1] J.E. Huber and N.A. Fleck, Multi-axial electrical switching of a ferroelectric: theory versus experiment, J. Mech. Phys. Solids, 49, 785-811, 2001

[2] J.E. Huber, N.A. Fleck, C.M. Landis and R.M. McMeeking, A constitutive model for ferroelectric polycrystals, J. Mech. Phys. Solids, 47, 1663-1697 (1999)

[3] M. Kamlah, Ferroelectric and ferroelastic piezoceramics-modelling of electromechanical hysteresis phenomena, Continuum Mech.Thermodyn., 13, 219-268 (2001)

[4] M. Kamlah and Q. Jiang, A model for PZT ceramics under uni-axial loading, Forschungszentrum Karlsruhe, FZKA 6211, 1998

[5] M. Kamlah and Z. Wang, A thermodynamically and microscopically motivated constitutive model for piezoceramics, Comput. Materials Science, 28, 409-418, (2003)

[6] G.A. Maugin and E. Bassiouny, Continuum thermodynamics of electromechanical hysteresis in ceramics, Continuum mechanics and its applications, 225-235, Hemisphere, New York (1989)

[7] R. McMeeking and C. Landis, A phenomenological multi-axial constitutive law for switching in polycrystalline ferroelectric ceramics, Internat. J. Engrg. Sci, 40, 1553-1557 (2002)

[8] H. Romanowski and J. Schröder, Modelling of the nonlinear ferroelectric hysteresis within a thermodynamically consistent framework, Proceedings of STAMM 2004, Darmstadt, to appear

[9] Y. Shu and K. Bhattacharya, Domain Patterns and Macroscopic Behavior of Ferroelectric Materials, Phil Mag. B, 81, 2021-2054 (2001)

[10] G. Francfort and A. Mielke, Existence results for a class of rate-independent material models with non-convex elastic energies, J. reine angew. Math., to appear

[11] A. Mielke and F. Theil, On rate-independent hysteresis models, Nonl. Diff. Eqns. Appl.(NoDEA), 11, 151-189 (2004)

[12] A. Mainik and A. Mielke, Existence results for energetic models for rate-independent systems, Calc. Var. PDSs, 22, 73-99 (2005)

[13] A. Mielke, Evolution in rate-independent systems, Handbook of Differential Equations II, Evolutionary Equations, Elsevier B.V., 2, 461-559, (2005)

[14] A. Mielke and F.Theil, A mathematical model for rate-independent phase transformations with hysteresis, Proceedings of the Workshop on "Models of Continuum Mechanics in Analysis and Engineering”, 117-129, Shaker-Verlag (1999)

[15] A. Mielke, F. Theil and V. Levitas, A variational formulation of rate-independent phase transformations using an extremum principle, Arch. Rational Mech. Anal., 162, 137-177 (2002)

[16] J. Fousek, Applications of Ferroelectrics, Proceedings of the Ninth IEEE International Symposium, 1-5 (1991)

[17] R. Temam, Navier-Stokes equations, volume 2 of Studies in Mathematics and its Applications, North-Holland Publishing Co., Amsterdam, third edition, 1984 